\title{
Fulminating panophthalmitis due to exogenous infection with Bacillus cereus: report of 4 cases
}

\author{
PATRICK C. HO, DENIS M. O'DAY, AND W. STEVEN HEAD \\ From the Department of Ophthalmology, Vanderbilt University School of Medicine, Nashville, Tennessee, \\ USA
}

SUMMARY Bacillus cereus is a seldom recognised but important cause of panophthalmitis. Although most reported cases have been the result of endogenous infection, we have recently seen 4 cases that followed ocular trauma. In each instance a fulminating panophthalmitis developed, accompanied by fever and leucocytosis. Despite prompt therapy all eyes were rapidly lost. In view of the selective antibiotic sensitivities of this organism and the fulminating course of the disease, conventional approaches to therapy, including standard prophylactic antibiotic regimens, are unlikely to be successful in such cases. Analysis of our cases suggest that ocular infection with $B$. cereus has certain features that may allow a provisional diagnosis to be made before isolation of the organism. Early recognition and prompt institution of effective therapy may lead to salvage of these eyes in the future.

Bacillus cereus is an unusual ocular pathogen. Haematogenous dissemination, usually in drug abusers, has been the route of infection in almost every reported case. ${ }^{1-5}$ Only 2 instances of exogenous infection have been reported in the past 30 years. ${ }^{56}$ However, we believe the reports may not accurately reflect the incidence of exogenous infection by this microorganism. During 1976-9 we treated 4 cases of traumatic panophthalmitis caused by $B$. cereus.

\section{Case reports}

CASE 1

A 33-year-old healthy Caucasian farmer presented to the Vanderbilt University Eye Clinic in severe ocular pain. Seventeen hours earlier a piece of metal entered his right eye while he was striking a metal cornpicker with a hammer. Vision was reduced to light perception, the lids were swollen and ecchymotic, and the conjunctiva was severely chemotic. On slit-lamp examination the cornea was diffusely oedematous, with both iris and vitreous prolapsing through a $2 \mathrm{~mm}$ scleral wound adjacent to the limbus. The anterior chamber was flat, and fundus details could not be visualised. A foreign body was localised outside the globe by radiography. Although the patient was

Correspondence to Denis M. O'Day, MD, Department of Ophthalmology, Vanderbilt University Medical Center, North, Nashville, TN, 37232, USA. afebrile, his white cell count was $14 \times 10^{9} / 1$. Antibiotic therapy with intravenous gentamicin $(3 \mathrm{mg} / \mathrm{kg} /$ day $)$ and methicillin $(80 \mathrm{mg} / \mathrm{kg} /$ day $)$ was begun. After excision of the prolapsed tissue the wound was repaired. $20 \mathrm{mg}$ of gentamicin and $40 \mathrm{mg}$ of dexamethasone were injected subconjunctivally.

Postoperatively the patient denied light perception in the injured eye. He became febrile $\left(100 \cdot 4^{\circ} \mathrm{F}, 38^{\circ} \mathrm{C}\right)$. The eye was markedly proptosed and a peripheral corneal ring abscess had developed. Large Grampositive rods and Gram-positive cocci were seen in a Gram stain from a conjunctival smear, raising the possibility of a clostridial infection. Fortified gentamicin $(13.5 \mathrm{mg} / \mathrm{ml})$ and 'fortified' bacitracin $(10000 \mathrm{U} / \mathrm{ml})$ were given topically every hour, and aqueous penicillin $(250000 \mathrm{U} / \mathrm{kg} /$ day $)$ together with gentamicin and methicillin were administered intravenously daily. However, worsening proptosis and extreme pain necessitated evisceration 12 hours later. Therapy was continued 4 more days, and the patient recovered uneventfully. Bacillus cereus was isolated from prolapsed tissue and cornea at the time of the initial operation and from the ocular contents removed at evisceration. Cultures from the corneal wound and prolapsed tissue also yielded enterococci.

\section{CASE 2}

A 43-year-old Caucasian labourer was hammering an axe head in his barn when a metal fragment entered 
his left eye. Twenty-four hours later he presented to Vanderbilt University Eye Clinic with a severe proptosis and conjunctival chemosis. A peripheral ring of oedema was visible in the cornea of the injured eye. Computerised axial tomography (CT scan) revealed the presence of a metallic intraocular foreign body in the posterior sclera of the left eye, but it could not be seen owing to the presence of massive vitreous haemorrhage. Although he was afebrile his white cell count was $16 \times 10^{9} / 1$. Vision was reduced to light perception. Intravenous therapy with gentamicin (4 $\mathrm{mg} / \mathrm{kg} / \mathrm{day})$ and methicillin $(80 \mathrm{mg} / \mathrm{kg} / \mathrm{day})$ was begun. At surgery prolapsed retina and vitreous were excised. The foreign body was extracted through the entry wound $7 \mathrm{~mm}$ posterior to the superior limbus, and $250 \mu \mathrm{g}$ of gentamicin was injected into the vitreous cavity. During the operation the surgeon observed the rapid development of a ring-shaped abscess in the area of the previously noted corneal oedema.

Postoperatively the patient's temperature rose to $100 \cdot 4^{\circ} \mathrm{F}\left(38^{\circ} \mathrm{C}\right)$. He was unable to perceive light. Proptosis became extreme and the abscess spread to involve the entire cornea. A culture of the scleral wound taken at surgery yielded Bacillus sp. and a coagulase negative staphylococcus. Intravenous antibiotic therapy with gentamicin and methicillin was continued, but 24 hours after the primary wound repair the eye was eviscerated because of severe pain. Intravenous chloramphenicol $(50 \mathrm{mg} / \mathrm{kg} /$ day $)$ was given postoperatively for 5 days. The patient became afebrile within 24 hours, and his white cell count returned to normal. Bacillus cereus was isolated from the eviscerated ocular contents.

\section{CASE 3}

A piece of metal entered the left eye of a healthy 6-year-old boy watching his father strike a hatchet with a hammer in the workshop of their farm. The next day, when he presented to the eye clinic at Vanderbilt Hospital, he had a fever of $100 \cdot 8^{\circ} \mathrm{F}$ $\left(38 \cdot 2^{\circ} \mathrm{C}\right)$ and a white cell count of $24 \cdot 5 \times 10^{9} / 1$. He was in severe pain and vision was reduced to light perception in the injured eye. The conjunctiva was chemotic and a ring infiltrate was present in the peripheral cornea. The fundus could not be seen owing to the presence of a large blood-stained hypopyon. Radiography of the left orbit revealed a metallic foreign body within the eye. After conjunctival cultures were taken, intravenous gentamicin $(5 \mathrm{mg} / \mathrm{kg} /$ day $)$ and methicillin $(100 \mathrm{mg} / \mathrm{kg} / \mathrm{day})$ were begun. Approximately 40 hours after the injury a large magnetic foreign body was removed through the scleral laceration $6 \mathrm{~mm}$ posterior to the limbus. After debridement of prolapsed necrotic retina and vitreous the wound was closed and a Silastic sponge was sewn into place. Gram-positive rods were seen in a smear of the excised retina and vitreous. Since infection with Clostridium perfringens was possible, intravenous penicillin $(250000 \mathrm{U} / \mathrm{kg} /$ day $)$ was administered postoperatively in addition to the other antibiotics. 'Fortified' bacitracin $(10000 \mathrm{U} / \mathrm{ml})$ and 'fortified' gentamicin $(13.6 \mathrm{mg} / \mathrm{ml})$ were given topically every hour.

Postoperatively the eye was blind. Proptosis worsened markedly with conjunctiva prolapsing through the lids. The cornea was totally opaque and necrotic. Twenty-four hours after surgery, because of increasingly severe pain, the eye was eviscerated. Intravenous antibiotic therapy, with the addition of chloramphenicol $(50 \mathrm{mg} / \mathrm{kg} / \mathrm{day})$, was continued for 5 days, and the patient recovered uneventfully. Bacillus cereus was isolated in pure culture from the eviscerated ocular contents. Conjunctival cultures taken at the time of initial examination, and cultures of the scleral wound taken at the time of primary repair, revealed a heavy growth of $B$. cereus.

\section{CASE 4}

A 28-year-old plumber was hit in the right eye by a piece of metal while he was hammering a steel bolt. He was in severe pain, and the visual acuity in the injured eye was reduced to $6 / 60$ when he presented to the eye clinic 7 hours after the injury. The cornea appeared normal, but the sclera was lacerated superiorly $3 \mathrm{~mm}$ from the limbus, with iris and vitreous prolapse. A moderate cellular reaction was present in the anterior chamber, and the pupil was peaked superiorly. He was afebrile and had a normal white cell count. An intraocular foreign body was demonstrated by radiography. Therapy with intravenous gentamicin $(5 \mathrm{mg} / \mathrm{kg} /$ day $)$ and methicillin (100 $\mathrm{mg} / \mathrm{kg} / \mathrm{day}$ ) was immediatey begun. Three hours later, after an unsuccessful attempt at removal of a foreign body, the prolapsed tissue was excised and the laceration repaired. Intravenous antibiotics were continued postoperatively.

About 36 hours after surgery his temperature rose to $100 \cdot 8^{\circ} \mathrm{F}\left(38 \cdot 2^{\circ} \mathrm{C}\right)$ and his white cell count was $19 \times 10^{9} / 1$. The eye was blind and he was in severe pain. A ring abscess had developed in the peripheral cornea. Ten hours later lid swelling and proptosis of the right globe became so severe that a lateral canthotomy was necessary. Fifty-eight hours after the injury the eye was eviscerated. Intravenous chloramphenicol $(60 \mathrm{mg} / \mathrm{kg} / \mathrm{day})$ was given with gentamicin for 5 days postoperatively and recovery was uneventful. Bacillus cereus was isolated from the intraocular contents. The wound cultures taken at the time of primary repair yielded $B$. cereus and a coagulasenegative staphylococcus. 
LABORATORY STUDIES

Bacillus cereus was isolated separately from eviscerated ocular contents and the entry wound in all 4 cases, from one extracted foreign body, and from the conjunctiva at the time of presentation in one case. In 3 patients a second organism was also present: coagulase-negative staphylococcus in 2 eyes and enterococcus in a third. Each Bacillus sp. was initially identified by Gram stain morphology, with final differentiation by biochemical testing. All isolates were resistant to penicillin, the semisynthetic penicillins, and the cephalosporins but sensitive to gentamicin, clindamycin, vancomycin, and chloramphenicol by the Kirby-Bauer disc diffusion method for in-vitro susceptibility testing.

\section{Discussion}

The aerobic Gram-positive bacilli are large endospore- forming rods. Until recently these microorganisms, with the exception of Bacillus anthracis, have been considered to be only mildly pathogenic for man. However, their involvement in a number of serious infections, including bacteraemia, endocarditis, meningitis, and food poisoning is now accepted. ${ }^{4-10}$ Since 1890 many cases of panophthalmitis attributed to Bacillus sp. have been recorded," and in 1934 François emphasised the destructive nature of Bacillus infection in a paper reporting 41 cases that followed trauma. ${ }^{12}$ Because of uncertainty over classification of this group of microorganisms, precise identification of isolates was seldom successful. In 1952 Davenport and Smith ${ }^{6}$ described the first culture-proved case of panophthalmitis caused by $B$. cereus, and since then the importance of this micro-organism in blood-borne infections has been emphasised in a number of papers. ${ }^{1-5}$ However, only 2 cases of exogenous ocular infection have been reported; both followed penetrating trauma. ${ }^{56}$

The clinical features of all our cases were remarkably similar. Three of the injuries occurred in an agricultural or rural setting; the fourth patient was at home using a hammer on a metal bolt. The lowvelocity metallic projectiles entered the eye at the limbus or anterior sclera, causing prolapse of vitreous into the conjunctival sac. Three foreign bodies were retained with the eyes; the fourth had its exit through the posterior sclera. Except for one patient who was examined within 7 hours of the injury, vision was reduced to light perception at the time of presentation. Subsequently vision in all eyes deteriorated rapidly to no light perception. Initially the severity of reactive orbital cellulitis, as indicated by proptosis, lid swelling, and loss of ocular movement, was variable. As the infection advanced, however, these signs dominated the clinical picture and in one case were extreme enough to require emergency canthotomy.

Progressive corneal changes appear to be a feature of this infection. Characteristically a ring of oedema adjacent to the limbus developed 24 to 36 hours after the injury. Within another few hours this area became infiltrated with inflammatory cells, and by 30 to 60 hours a ring abscess was evident. This distinctive finding has also been reported in endogenous cases. ${ }^{235}$

Standard management of the injuries proved to be ineffective. Despite debridement, closure of the entry wounds, foreign body removal, and comprehensive antibiotic therapy the infections progressed inexorably, accompanied by fever and a moderate leucocytosis. Evisceration was necessary within 2 to 3 days of the injury, and thereafter the systemic reaction abated promptly. In each case infection with $B$. cereus was confirmed by recovery of the micro-organism from the eviscerated ocular contents; in 3 cases a second organism was also present.

Bacillus species are present in air, soil, water, milk, dust, wool, and faeces. Since the injuries in all cases occurred in a setting where these micro-organisms could have contaminated the tools being used, it is likely that the low-velocity foreign bodies carried bacteria into the eye. However, this explanation may not apply to all cases. Although penetrating injuries due to low-velocity projectiles are frequent, this devastating infection occurs only rarely, suggesting that other mechanisms need to be considered. In each of our cases vitreous prolapse was present and may have provided a portal of entry for organisms already present in the conjunctiva. Although B. cereus is not a usual inhabitant of the conjunctival sac, Van Bijsterveld and Richards ${ }^{13}$ showed that it may become part of the flora in the appropriate environment. They isolated $B$. cereus from 40 eyes of asymptomatic, infection-free individuals engaged in the processing and handling of hay or the care of stables and horses at a race track. It is therefore of interest that $B$. cereus was recovered from the conjunctiva of one of our patients (case 3) at the initial examination.

Once the micro-organism gains access to the vitreous, infection appears to progress with remarkable speed. It is evident that panophthalmitis is established within 48 hours of injury. This unusually fulminating course appears to be characteristic of $B$. cereus infection and distinguishes this micro-organism from the other Gram-positive aerobic bacilli. While it is not clear why $B$. cereus should be so devastating to the eye, it seems likely that exotoxin production plays a part. ${ }^{9}$ Bacillus cereus is known to elaborate a number of exotoxins, including phospholipase $\mathrm{C}$, haemolysin, several proteases, and an enterotoxin. ${ }^{14}$ Although the ocular effects of these metabolites have yet to be investigated, each has the potential to inflict 
direct tissue damage. The enterotoxin, which has both dermonecrotic and vascular permeability effects, has been shown to be the cause of diarrhoea in $B$. cereus food poisoning. ${ }^{15}$

The loss of every eye infected with $B$. cereus reported thus far highlights the limitations of present therapeutic approaches. The isolates from our cases were resistant to natural penicillins, the semisynthetic penicillins, and the cephalosporins. They were susceptible to gentamicin, one of the antibiotics routinely used following penetrating ocular trauma. The inability of gentamicin to control the infection in our cases illustrates the need to develop fresh approaches to treatment. Studies in our laboratory are now in progress evaluating several other antibiotics, including clindamycin and vancomycin. Clindamycin alone or in combination with other antibiotics may prove to be a useful antibiotic for treatment of this infection in view of its excellent ocular penetration. ${ }^{16}$

This work was supported in part by NIH grant EY 01621 and an unrestricted grant from Prevention of Blindness, Inc.

\section{References}

1 Kerkenezov N. Panophthalmitis after a blood transfusion. Br J Ophthalmol 1953; 37: 632-6.

2 Hatem G, Merritt JC, Cowan CL. Bacillus cereus panophthalmitis after intravenous heroin. Ann Ophthalmol 1979; 11:431-40.
3 Bouza E, Grant S, Jordan MC. Bacillus cereus endogenous panophthalmitis. Arch Ophthalmol 1979; 97: 498-9.

4 Tuazon CU, Murray HW, Levy C, Solny MN, Curtin JA, Sheagren JN. Serious infections from Bacillus species. JAMA 1979; 241: 1137-40.

5 Young EJ, Wallace RJ, Ericsson CD, Harris RA, Claridge J. Panophthalmitis due to Bacillus cereus. Arch Int Med 1980; 140: 555-60.

6 Davenport R, Smith C. Panophthalmitis due to an organism of the Bacillus subtilis group. Br J Ophthalmol 1952; 36: 389-92.

7 Ihde DC, Armstrong D. Clinical spectrum of infection due to Bacillus species. Am J Med 1973; 55: 839-45.

8 Farrar WE. Serious infection due to 'non-pathogenic' organisms of the genus Bacillus. Am J Med 1963; 34: 134-41.

9 Turnbull PCB, Jorgensen K, Kramer JM, Gilbert RJ, Parry JM. Severe clinical conditions associated with Bacillus cereus and the apparent involvement of exotoxins. J Clin Pathol 1979; 32: 289-93.

10 Turnbull PCB, French TA, Dowsett EG. Severe systemic and pyogenic infections with Bacillus cereus. Br Med J 1977; i: 1628-33.

11 Poplawska S. Zur Aetiologie der Panophthalmie nach Verletzung durch Fremdkorper. Fortschr Med 1890; 8: 489-92.

12 François JM. Le bacille subtilique en pathologie oculaire. Bull Soc Ophtalmol Fr 1934; 47: 423-6.

13 Van Bijsterveld OP, Richards RD. Bacillus infections of the cornea. Arch Ophthalmol 1965; 74: 91-5.

14 Gordon R. Endospore-forming rods and cocci. In: Buchanan RE, Gibbons NE, eds. Bergey's Manual of Determinative Bacteriology. 8th ed. Baltimore: Williams and Wilkins, 1974: $529-49$.

15 Turnbull PCB, Kramer JM, Jorgensen K, Gilbert RJ, Melling J. Properties and production characteristics of vomiting diarrheal and necrotizing toxins of $B$. cereus. Am J Clin Nutr 1979; 32: $219-28$.

16 Tabbara KF, O'Connor GF. Ocular tissue absorption of clindamycin phosphate. Arch Ophthalmol 1975; 93: 1180-5. 